TI 2013-183/I

Tinbergen Institute Discussion Paper

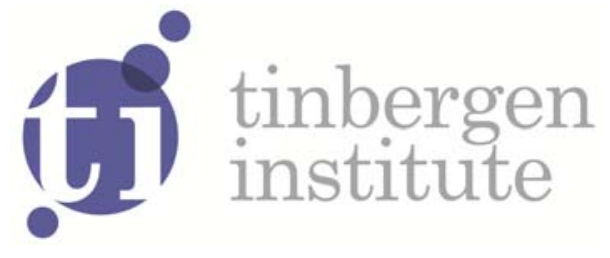

\title{
Individual Team Productivity - A Conceptual Approach
}

Julia Mül/er

Thorsten Upmann²

Joachim Prinz²

' Erasmus School of Economics, Erasmus University Rotterdam, and Tinbergen Institute, The Netherlands;

2 University Duisburg-Essen, Germany. 
Tinbergen Institute is the graduate school and research institute in economics of Erasmus University Rotterdam, the University of Amsterdam and VU University Amsterdam.

More TI discussion papers can be downloaded at http://www.tinbergen.nl

Tinbergen Institute has two locations:

Tinbergen Institute Amsterdam

Gustav Mahlerplein 117

1082 MS Amsterdam

The Netherlands

Tel.: +31(0)205251600

Tinbergen Institute Rotterdam

Burg. Oudlaan 50

3062 PA Rotterdam

The Netherlands

Tel.: +31(0)10 4088900

Fax: $+31(0) 104089031$

Duisenberg school of finance is a collaboration of the Dutch financial sector and universities, with the ambition to support innovative research and offer top quality academic education in core areas of finance.

DSF research papers can be downloaded at: http://www.dsf.nl/

Duisenberg school of finance

Gustav Mahlerplein 117

1082 MS Amsterdam

The Netherlands

Tel.: +31(0)20 5258579 


\title{
Individual Team Productivity - A Conceptual Approach
}

Julia Müller*
Thorsten Upmann ${ }^{\dagger}$

Joachim Prinz

November 4, 2013

\begin{abstract}
Teams, in both firms and in sports, jointly produce a product. While a fixed task is assigned to each member of a team, the individual team productivity of a worker or player is difficult to conceptualize. This is particularly true, if this concept is aimed to be operable on observable data. In this paper we, therefore, propose two versions of a new concept of individual team productivity which is closely related to eigenvalue centrality; accordingly we refer to it as eigenvalue productivity. For each version of eigenvalue productivity we provide an example demonstrating the operability of our concept.
\end{abstract}

Keywords: individual team productivity; eigenvalue productivity; centrality; team production; team sports

JEL Classification: D24, J24, L23, L83

Acknowledgments: The authors are grateful to Oliver Gürtler for valuable comments and suggestions. We also thank the participants of the EALE conference 2013 for helpful discussion.

${ }^{*}$ Erasmus University Rotterdam \& Tinbergen Institute, Post Box 1738, 3000 DR Rotterdam, Netherlands. Email: JMuller@ese.eur.nl

${ }^{\dagger}$ University Duisburg-Essen, Mercator School of Management, Lotharstraße 65, 47057 Duisburg, Germany. Email: Thorsten.Upmann@uni-duisburg-essen.de

${ }^{\ddagger}$ University Duisburg-Essen, Mercator School of Management, Lotharstraße 65, 47057 Duisburg, Germany. Email: Joachim.Prinz@uni-duisburg-essen.de 


\section{INTRODUCTION}

A team is more than merely the sum of its members' individual abilities - and this is true for teams in economics and in sports likewise. Co-workers interact and their abilities as well as their capacities for and their productivities in teamwork determine, among other factors, the actual team output - and thus the success of the team. In this way, the interplay of a worker with his team-mates depends on the ability and willingness of the other co-workers to co-operate: A worker can only interact with his team-mates efficaciously, if these allow for this. As a consequence, effective team play of a worker depends on the team play and thus on the cooperative capacity and on the effort of the other co-workers. ${ }^{1}$

While this appraisal should be beyond controversy, it does not readily provide an operational concept of individual capacity of team productivity. Similarly, coworkers' team productivities can not directly observed and are thus not readily available from the team data. This puts forth the following questions: How can we (i) consistently define and then (ii) calculate the individual team productivities from available data. While the productivity of a team is relatively easy to measure, e. g. by total sales, revenue, or patterns, by number of orders or in team sports by win percentage, score points, goals etc., it is disproportionately more difficult to measure the productivity of an individual player within a team. This is arguably a consequence of the fact that "the interaction between team members is multifaceted" (Depken and Haglund, 2011, p. 4).

For example in team sports the literature has used various variables to appraise the individual team productivity of a player: the number goals shot, assists provided, duels won, ball touches etc. Yet, all of these numbers suffer from the fact that they impute an output (successful action) to an individual player, while this output is actually the joint product of the player and his team-mates: A player can only perform nicely, when his team-mates are able and willing to put him into scene and lay the proper ground for his actions to become fruitful. At the same time, professional team sport requires, besides individual aptitudes and skills, to a high degree complex collective moves, which represent both the prerequisite for and the consequence of individual performance (see, for example, also Beck and Meyer, 2012). Through all of these channels the team productivity of a player depends on the productivities of his team-mates, and in particular on the productivity of his

\footnotetext{
${ }^{1}$ The relevance of effort choices of workers within teams (based on income maximizing considerations) has recently been emphasized by Gould and Winter (2009).
} 
"neighbours" on the playing field. ${ }^{2}$ And similar arguments apply to any complex team production process in economics.

The empirical literature on teams in both firms and team sports focuses on individual productivity, team productivity, wages and wage distributions. The empirical significance of spillovers to productivity across players/co-workers within teams has been demonstrated in various domains, most recently by, among others, Idson and Kahane (2000, 2004), Kendall (2003), Mas and Moretti (2009), Franck and Nüesch (2010) and Alvarez et al. (2011). And even though, the productivity of a worker/player may significantly differ across different firms/teams, the relevance of co-worker and team-mate effects on individual productivity is apparently an omnipresent phenomenon; and the relevance of this team composition has most recently been emphasized by Beck and Meyer (2012). ${ }^{3}$

In order to capture the prevalence of spillover effects and the interdependency of team productivities among co-workers, a more sophisticated concept to measure the contribution (or the relevance) of a co-worker to the productivity of his team seems to be necessary. In this paper we, therefore, delineate two variants of a concept of individual team productivity, which makes use of the self-referential structure of co-operation within teams: In team co-workers are required to co-operate in order to make the team perform successfully, and each co-worker benefits from the co-operative abilities of his team-mates. The more a team-mate of co-worker $i$ contributes to the team, the better the conditions for co-worker $i$ to perform well and thus to contribute to the success of the team. In this way the team productivity of co-worker $i$ depends (positively) on the productivities of his team-mates, and in particular on the productivity those who are "adjacent to" or "central for" him. Since this holds true for any co-worker, team productivity is self-referential and as a consequence, the team productivities of all co-workers of a team must be determined simultaneously.

The measurement of the productivity of an individual player participating in a team for the specific example of a basketball team of the NBA was done by Berri (1999). He proposed an econometric model tailored for basketball. Also, he emphasizes that the sometimes used seemingly straightforward shortcuts for individual

\footnotetext{
${ }^{2}$ Accordingly, there is empirical evidence that some combinations of positions or some pairs of players are more strongly complementary than others (see, for example, Idson and Kahane, 2000).

${ }^{3} \mathrm{Also}$, there is large interest in the relationship between salaries, namely wage disparity within the team, and team performance, which has been explored by, for example, Depken (2000), Simmons and Berri (2011).
} 
team productivity like number of points scored are "likely to be incorrect and misleading" (p.415), and that those shortcuts neglect the team-sport aspect, i. e.the co-operation within a team we discussed in the preceding paragraph. In contrast, to the work of Berri (1999) our concept (with its two versions) is quite general and flexible enough to cope with many different economic contexts and available data bases - and is thus applicable in many situations.

We duly acknowledge the feature of mutual complementarity in production ${ }^{4}$ within teams and present two variants of a new concept of individual team productivity, both of which consistently and simultaneously define the contributions of each co-worker to the productivities of all other team-mates. In this way, the two variants of the concept serve to measure the co-operative productivity - and thus the importance or centrality — of a co-worker within a team. Formally, both versions are closely related to the concept of eigenvector centrality, which is known in network analysis and has been suggested by Bonacich (1972). ${ }^{5}$ While mathematically very similar, both variants differ with respect to the data required to calculate the individual team productivities of the co-workers.

We demonstrate this by means of examples taken from team sports. While the first variant requires only the data for the sportive results of a team during a season and the respective line-ups, the second makes use of detailed data on directed passes played in one game between any pair of players within a team. Since the data for team results and line-ups is readily available, the first concept is arguably broadly applicable in team sports. The second concept, though, is employable even if a team has played very few games, or even a single one, provided that detailed pass statistics are available. Similar arguments apply for team production in economic environments.

We are thus confident that our variants of the concept of individual team productivity do not only constitute a significant theoretical contribution, but may help calculate individual team productivities in applied empirical work. In this way

\footnotetext{
${ }^{4}$ Formally, complementarity (in production) is captured by supermodularity of (production) functions. For more details see, for example, Milgrom and Roberts (1990) or the excellent monograph of Topkis (1998).

${ }^{5}$ The idea can even be traced back to Katz (1953) who proposed a similar index to measure the status of individuals within a group. (For a textbook presentation of centrality indices see, for example, Jackson, 2008, sec. 2.2.4.)
} 
our work obeys a suggestion of Kendall (2003) who called for more research "to derive measures of players' 'true' marginal products when productivity spillovers exist" (p. 401).

The rest of the paper is structured as follows. In Section 2 we introduce our general approach to conceptually formalize team productivity. Our first concept of individual team productivity, between eigenvalue productivity (between EVP), is the presented in Section 3, and illustrated by an example in Section 4. We then define a closely related variant of individual team productivity, within eigenvalue productivity (within EVP), in Section 5, which is illustrated by means of the data of a game from the 2010 FIFA World Cup. Finally, in Section 6 we summarize our results and conclude.

\section{The Approach}

We present two different concepts of individual team productivity or co-worker productivity and show how theses individual productivities may be consistently determined for all members of a team. In this chapter we present our notation using for illustrative purposes first and foremost the example of team sports. Yet our concepts are quite general and can be applied to almost any working environment with joint production. Accordingly, the reader may in the following substitute player by co-worker, football team by team, squad by personnel, etc.

Consider a team consisting of a fixed set of $n$ players $N=\{1,2, \ldots, n\}$, in team sports this is the squad of the team. For example, for a football team the squad must consist of at least eleven players, and should for convenience actually exceed this minimum requirement. ${ }^{6}$ A line-up is then a subset of the squad, $T \subseteq N$, such that $|T|$ equals the required number of players on the field (or workers on a project).

As argued above, a player benefits from the abilities of his team-mates: The more a team-mate of player $i$ contributes to the team, the better the conditions for player $i$ to perform well and thus to contribute to the success of the team. In this way the team productivity of player $i$ depends (positively) on the productivities of his team-mates. We shall now formalize this idea and show how the individual team productivities may be derived.

\footnotetext{
${ }^{6}$ In fact the squad of football teams in the German Bundesliga amounts to roughly 25 to 30 players.
} 
Let the team productivity of player $i$ in team $N$ be denoted by $p^{i}(N)$; and the vector of team productivities of the players of the team by $\mathbf{p}(N), \mathbf{p}(N) \in \mathbb{R}_{+}^{n} \cdot{ }^{7}$ We are thus assuming that the individual productivity of each player is non-negative. According to our argument provided above, we presume that the productivity depends linearly on the productivity of his or her team-mates. We thus write the individual team productivity of player $i$ in team $N$ as

$$
p^{i}(N)=\frac{1}{\lambda} \sum_{j \in N, j \neq i} g_{i j}(N) p^{j}(N) \quad \forall i \in N,
$$

where $g_{i j}(N) \in[0,1]$, denotes the extent to which player $i$ benefits from the individual team productivity of player $j$ (when both belong to team $N$ ), and $\lambda \in \mathbb{R}_{+} \backslash\{0\}$, represents some (strictly positive) factor of normalization. (For convenience we define $g_{i i}(N)=0$ for all $i \in N$, implying that player $i$ may not enhance his or her own productivity by multiplicator process.) In this way, the individual team productivity of player $i$ depends on the individual team productivity of his team-mates, but not on his or her own; and since this relation holds for any player $i \in N$, we arrive at (in matrix notation)

$$
\mathbf{p}(N)=\frac{1}{\lambda} \mathbf{G}(N) \mathbf{p}(N),
$$

where $\mathbf{G}(N) \equiv\left[g_{i j}(N)\right]_{i, j \in N}$ is the matrix of the coefficients measuring the extent to which the team productivities influence each other within the team. Subsequently we suppress the team argument $N$ for notational convenience, but it should be kept in mind that the $\mathbf{G}$ and $\mathbf{p}$ depend on the team under consideration. Then, equation system (1) may equivalently be written as

$$
\lambda \mathbf{p}=\mathbf{G} \mathbf{p} \quad \Leftrightarrow \quad(\mathbf{G}-\lambda \mathbf{I}) \mathbf{p}=0
$$

where $\mathbf{I}$ denotes the identity matrix (of the proper rank, i. e. of rank $n$ in this case). For $\mathbf{p} \neq \mathbf{0}$, the homogeneous equation system (2) has a solution (in $\mathbf{p}$ ) if, and only if,

$$
\operatorname{det}(\mathbf{G}-\lambda \mathbf{I})=0
$$

But this is equivalent with $\lambda$ being an eigenvalue of $\mathbf{G}$, and $\mathbf{p}$ being the corresponding eigenvector. ${ }^{8}$ Since $\mathbf{p}$ is the vector of individual team productivities - which we want

\footnotetext{
${ }^{7}$ The set $\mathbb{R}_{+}^{n}:=\left\{x \in \mathbb{R}^{n}: x \geq 0\right\}$ denotes the non-negative orthant of $\mathbb{R}^{n}$, where we define for any $x, y \in \mathbb{R}^{n}: x \geq y: \Leftrightarrow x_{i} \geq y_{i} \forall i=1, \ldots, n$.

${ }^{8}$ The eigenvalues are the solutions of the characteristic polynomial equation (of degree $n$ ) resulting from equation (3).
} 
to determine - we refer to our concept of individual team productivities as eigenvalue productivity (EVP).

Since individual team productivities are, by assumption, non-negative, and we assume that there is at least one player whose team productivity is positive, we are only interested in eigenvectors $\mathbf{p} \in \mathbb{R}_{+}^{n} \backslash\{0\}$. Fortunately, the matrix $\mathbf{G}$ is non-negative so that we may apply the Perron-Frobenius theorem for non-negative, indecomposable matrices (provided that is $\mathbf{G}$ indecomposable).$^{9}$ Roughly, this theorem says that there is a positive eigenvalue $\hat{\lambda}$ of $\mathbf{G}$, this eigenvalue is a simple eigenvalue, all other eigenvalues are absolutely smaller than $\hat{\lambda}$, and, most importantly, all components of the eigenvector associated with $\hat{\lambda}$ are positive. ${ }^{10}$ For this reason we are (only) interested in the largest real-valued eigenvalue of $\mathbf{G}$, i. e. in $\hat{\lambda}$, and in the associated eigenvalue.

Given matrix $\mathbf{G}$ it is thus straightforward to calculate the individual team productivities $(E V P s)$ of the players for a given team. Since frequently one cannot directly observe the (marginal) effect of player $j$ 's productivity on player $i$ 's productivity, the coefficient matrix $\mathbf{G}$ is not given, but its elements have to be calculated from the data. Having defined individual team productivity in terms of EVP, the problem thus boils down to calculating $\mathbf{G}$. Both of our two concepts of individual team productivity (presented in sections 3 and 5) rest upon the idea of employing eigenvalue productivity, but differ in the way $\mathbf{G}$ is calculated from data. The idea to provide two different concepts reflects our endeavor to provide an approach with is broadly applicable to different economic situations and therefore has to cope with the available data in that particular situation.

\section{Between Eigenvalue Productivity}

The kind of situation we cover with our first concept is a situation where the team has played during a season or a year with different line-ups. Accordingly, this concept utilises the different team compositions and their respective performance to calculate individual team productivity. The variance in the line-ups is therefore

\footnotetext{
${ }^{9}$ See, for example, Takayama (1985), p. 372, Theorem 4.B.1 (and p. 375, Theorem 4.B.2 for not indecomposable matrices); or alternatively Gantmacher (2000) or Horn and Johnson (1990).

(A squared matrix $\mathbf{A}$ is called decomposable if there exists a permutation matrix $\mathbf{P}$ such that $\mathbf{P}^{-1} \mathbf{A P}$ is an upper block triangular matrix, see Takayama, 1985, p. 370.)

${ }^{10} \mathrm{Only}$ if $\mathbf{G}$ is not indecomposable, may some elements (but not all) of the associated eigenvector be zero.
} 
essential to calculate the matrix G. Because we make use of the differences in performance between different line-ups, we name this concept between eigenvalue productivity (between EVP). We next demonstrate how to calculate $\mathbf{G}$.

To begin with, we have to delete from the squad of the team, i. e. from the set $N$, those players who have never played on the field (have never been in action) during the season. Then, we calculate the entries of $\mathbf{G}$ as follows. For each given pair ${ }^{11}$ of players $\{i, j\}, i, j \in N$, consider those matches in which $i$ and $j$ have played together, that is where both were included in the line-up; let $m_{i j}$ denote this number, and calculate the points the team has achieved in these matches relative to the maximal number of points the team could have achieved; let $s_{i j}$ denote this ratio of points, which measures the success (performance) of the pair $\{i, j\}$. Next consider those matches where player $i$ was a member of the line-up (player $j$ may or may not be a member of the line-up), and call this number $m_{i}$; calculate the points the team has achieved in these matches relative to the maximal number of points the team could have achieved; denote this ratio of points by $s_{i}$, which measures the success (performance) of player $i$.

Then $g_{i j}:=s_{i j} / s_{j}$ represents the success of the pair $\{i, j\}$ relative to the success of player $j$ - and this effect is arguably associated with the presence of player $i$. This allows us to define $\mathbf{G}$ by $\mathbf{G}:=\left(g_{i j}\right)_{i, j \in N}$. As a convention, for pairs of players $\{i, j\}$ that have never been jointly included in some line-up during the season we set $s_{i j}=s_{j}$ and thus $g_{i j}=1$. Observe that $\mathbf{G}$ is non-negative, and (generically) not symmetric.

\section{EXAMPLE}

We now use an hypothetical example of a small team and provide the necessary data to apply our first concept. The purpose of this simple example is to demonstrate the calculation of the proposed between eigenvalue productivity (between EVP).

Consider a team consisting of six players $N=\{A, B, C, D, E, F\}$. Assume that during the season the team has played 17 games in six different line-ups, and has won 26 out of 51 possible points. The detailed results for the specific line-ups are displayed in Table 1.

Apparently player $F$ was never included in a line-up during the season, so that his team productivity can neither be reasonably defined nor measured. Accordingly,

\footnotetext{
${ }^{11}$ In this context the word pair refers to an unordered pair, that is to a set containing two elements.
} 
we completely disregard player $F$ henceforth. Moreover, observe that players $A$ and $B$ were never included in the same line-up, a feature which may occur, for example, for players playing on the same position (e.g. goalkeepers).

\begin{tabular}{cccccc} 
no. & line-up & games & points & max. pts & ratio \\
\hline 1 & ACD & 2 & 3 & 6 & $\frac{1}{2}$ \\
2 & ACE & 4 & 12 & 12 & 1 \\
3 & ADE & 3 & 1 & 9 & $\frac{1}{9}$ \\
4 & BCD & 3 & 4 & 9 & $\frac{4}{9}$ \\
5 & BCE & 2 & 6 & 6 & 1 \\
6 & BDE & 3 & 0 & 9 & 0 \\
\hline & sum & 17 & 26 & 51 & $\frac{26}{51}$ \\
\hline
\end{tabular}

TABLE 1. Results of the team for varying line-ups

Before we proceed to calculate the between EVP of the single players, it is worthwhile to pause for a second and to inspect Table 1 for the individual contributions of the single players. Apparently, the success of the team has improved whenever player $C$ joined the team: compare line-up $A D E$ with $A C D, A D E$ with $A C E, B D E$ with $B C D$, and $B D E$ with $B C E$. In all of these comparisons the ratio of achieved to maximal points has gone up by substituting either player $D$ or player $E$ by player $C$. It thus appears that the individual team productivity of player $C$ is high — and this should be reflected by our measure of individual team productivity, the between EVP. Also, it is easy to verify that the team performance has improved whenever player $B$ has been substituted by player $A$; and that the team performance has declined whenever player $D$ has been included in a line-up. Accordingly, the between EVP should assign a higher individual productivity to player $A$ than to player $B$, and it should certify a particularly poor individual team productivity to player $D$. - We shall see that this is exactly the case.

From Table 1 we calculate the results for each single player $i$ by disregarding those games where player $i$ was not a member of the line-up. The individual results are shown in Table 2. In the next step we have to calculate the success for each pair of players $\{i, j\}, i, j \in N$, this is done in Table 3 . Then, the matrix of pair-wise 


\begin{tabular}{ccccc} 
player & incl. in line-ups & points & max. pts & $s_{i}$ \\
\hline$A$ & $\{1,2,3\}$ & 16 & 27 & $\frac{16}{27}$ \\
$B$ & $\{4,5,6\}$ & 10 & 24 & $\frac{5}{12}$ \\
$C$ & $\{1,2,4,5\}$ & 25 & 33 & $\frac{25}{33}$ \\
$D$ & $\{1,3,4,6\}$ & 8 & 33 & $\frac{8}{33}$ \\
$E$ & $\{2,3,5,6\}$ & 19 & 36 & $\frac{19}{36}$ \\
\hline
\end{tabular}

TABLE 2. Individual results for each player

\begin{tabular}{ccccc} 
pair & incl. in line-ups & points & max. pts & $s_{i j}$ \\
\hline$\{A, C\}$ & $\{1,2\}$ & 15 & 18 & $\frac{5}{6}$ \\
$\{A, D\}$ & $\{1,3\}$ & 4 & 15 & $\frac{4}{15}$ \\
$\{A, E\}$ & $\{2,3\}$ & 13 & 21 & $\frac{13}{21}$ \\
$\{B, C\}$ & $\{4,5\}$ & 10 & 15 & $\frac{2}{3}$ \\
$\{B, D\}$ & $\{4,6\}$ & 4 & 18 & $\frac{2}{9}$ \\
$\{B, E\}$ & $\{5,6\}$ & 6 & 15 & $\frac{2}{5}$ \\
$\{C, D\}$ & $\{1,4\}$ & 7 & 15 & $\frac{7}{15}$ \\
$\{C, E\}$ & $\{2,5\}$ & 18 & 18 & 1 \\
$\{D, E\}$ & $\{3,6\}$ & 1 & 18 & $\frac{1}{18}$ \\
\hline
\end{tabular}

TABLE 3. Pair results

success is given by

$$
\mathbf{S}=\left(s_{i j}\right)_{i, j \in N}=\left(\begin{array}{ccccc}
\frac{16}{27} & \frac{5}{12} & \frac{5}{6} & \frac{4}{15} & \frac{13}{21} \\
\frac{16}{27} & \frac{5}{12} & \frac{2}{3} & \frac{2}{9} & \frac{2}{5} \\
\frac{5}{6} & \frac{2}{3} & \frac{25}{33} & \frac{7}{15} & 1 \\
\frac{4}{15} & \frac{2}{9} & \frac{7}{15} & \frac{8}{33} & \frac{1}{18} \\
\frac{13}{21} & \frac{2}{5} & 1 & \frac{1}{18} & \frac{19}{36}
\end{array}\right)
$$

Note that in building $\mathbf{S}$ we have used the convention $s_{i i}:=s_{i}$ and $s_{i j}=s_{j}$ for pairs $\{i, j\}$ who have not been included in any line-up; which here applies to the pair $\{A, B\}$ only.

If we then relate the success of the pair $\{i, j\}$ to the success of player $i$, we obtain the ratio $g_{i j}:=s_{i j} / s_{j}$, representing the relative out-/under-performance of player $j$ due to the presence of player $i$. In this way we are able to build matrix G. Entries in matrix $\mathbf{G}$ relate the success of a pair of players to the presence of a 
single player. In the $j$ th column of matrix $\mathbf{G}$ the success of a pair is normalized by the presence of the same player $j$, while in the $i$ th row of matrix $\mathbf{G}$ the success of a pair is normalized by the presence of the different players 1 to $n$. In this way $\mathbf{G}$ represents all relative normalized pairwise success ratios.

$$
\mathbf{G}=\left(g_{i j}\right)_{i, j \in N}=\left(\begin{array}{ccccc}
1 & 1 & \frac{11}{10} & \frac{11}{10} & \frac{156}{133} \\
1 & 1 & \frac{22}{25} & \frac{11}{12} & \frac{72}{95} \\
\frac{45}{32} & \frac{8}{5} & 1 & \frac{77}{40} & \frac{36}{19} \\
\frac{9}{20} & \frac{8}{15} & \frac{77}{125} & 1 & \frac{2}{19} \\
\frac{117}{112} & \frac{24}{25} & \frac{33}{25} & \frac{11}{48} & 1
\end{array}\right) .
$$

By construction $g_{i i}=1$ for all $i \in N$, and $g_{i j}=1$ for all $i, j \in N$, who have not been playing in a joint line-up. Finally, we have to calculate the eigenvalues of $\mathbf{G}$, which are given by $\{4.9737,0.8661,-0.8584,0.0093+0.0978 i, 0.0093-0.0978 i\}$. Since the first eigenvalue is the largest one, we set $\lambda=4.9737$ and calculate the associated eigenvector: this is given by $\mathbf{p}_{\lambda}=(1.05419,0.88881,1.44473,0.489131,1$.$) - which$ is the vector we have been looking for: the vector of between $E V P$, the individual team productivities of the players. ${ }^{12}$ As expected, the between EVP of player $A$ and $C$ are high, while those of players $B$ and $D$ are low. Apparently the between $E V P$ reflects our intuitive notion of individual team productivity.

\section{Within Eigenvalue Productivity}

To be able to capture any kind of situation we present a second approach which can be used when the data is inadequate to calculate the between eigenvalue productivity. For example, if a team has played only few games such that the pair-wise success ratios would be based on very few observations, which may happen for teams taking part in championships such as the FIFA World Cup or the UEFA European Football Championship. Also, the data may be inadequate, if there appeared only little variability in the line-up such that the pair-wise success ratios would frequently coincide, which may happen for a team with a small squad and/or a fixed starting team. In all of these situations the approach presented in Section 3 appears to be unsuitable. In this section we therefore propose a second approach to measure the individual team productivity of each player when the team has played only few games — or even a single game.

\footnotetext{
${ }^{12}$ Note that we may normalise $\mathbf{p}_{\lambda}$ in any way which appears to be convenient: Thus, any multiplicative of $\mathbf{p}_{\lambda}: \mathbf{q}_{\lambda}=\alpha \mathbf{p}_{\lambda}, \alpha>0$ may also serve as a vector of team productivities.
} 


\begin{tabular}{rlrcl} 
no. & name & $\begin{array}{c}\text { playing } \\
\text { time }\end{array}$ & $\begin{array}{c}\text { passes } \\
\text { played }\end{array}$ & $\begin{array}{c}\text { notional } \\
\text { passes }\end{array}$ \\
\hline 1 & Romero & $0-90$ & 16 & 20.271 \\
2 & Demichelis & $0-90$ & 53 & 63.086 \\
3 & Burdisso & $0-90$ & 38 & 41.986 \\
4 & Heinze & $0-90$ & 47 & 74. \\
5 & Di Maria & $0-75$ & 33 & 40.629 \\
6 & Higuain & $0-90$ & 20 & 25.6 \\
7 & Messi & $0-90$ & 57 & 77.157 \\
8 & Tévez & $0-90$ & 46 & 66.957 \\
9 & Mascherano & $0-90$ & 59 & 68.629 \\
10 & Otamendi & $0-70$ & 42 & 55.8 \\
11 & Rodríguez & $0-90$ & 51 & 57.586 \\
12 & Aguero & $75-90$ & 5 & 30. \\
13 & Pastore & $70-90$ & 10 & 45. \\
\hline
\end{tabular}

TABLE 4. Line-up of the Argentinean team (Source: FAS research)

Suppose that for that case some measure of bilateral co-operation between any two team-mates in the course of a game is available. For example we may use for any pair $\{i, j\}$ the passes played from player $i$ to player $j$, and from player $j$ to player $i$. With this data we build up the matrix of passes played $\mathbf{G}$ (for a given game and a given line-up). Since this matrix is non-negative, we may proceed to calculate the largest eigenvalue of $\mathbf{G}$ and the associated eigenvector, just as indicated in Sections 2 and 3 .

In order to illustrate this idea, we apply an actual example: We use the quarterfinal of the 2010 FIFA World Cup between Argentina and Germany (0:4), Cape Town, South Africa, July 3, 2010. The line-up of the Argentinean team, the actual playing time for each player and the passes played by each player are given in Table 4 . Moreover, the last column of this table contains, what we call, the notional passes, which are passes extrapolated if all players had played over the full playing time of 90 minutes. (The details of this calculation will be explained below.) 
The detailed passes played between any two Argentinean players $i, j \in T=$ $\{1,2, \ldots, 13\}$ are given in matrix ${ }^{13}$

$$
\mathbf{G}=\left(g_{i j}\right)_{i, j \in T}=\left(\begin{array}{ccccccccccccc}
0 & 5 & 2 & 1 & 1 & 2 & 0 & 0 & 1 & 2 & 1 & 0 & 1 \\
2 & 0 & 9 & 1 & 4 & 5 & 6 & 1 & 9 & 8 & 6 & 0 & 2 \\
1 & 9 & 0 & 7 & 1 & 0 & 3 & 3 & 7 & 1 & 5 & 0 & 1 \\
0 & 0 & 5 & 0 & 5 & 3 & 7 & 9 & 7 & 0 & 4 & 1 & 6 \\
0 & 0 & 1 & 2 & 0 & 6 & 11 & 8 & 0 & 3 & 2 & 0 & 0 \\
0 & 4 & 0 & 0 & 3 & 0 & 5 & 1 & 2 & 0 & 4 & 1 & 0 \\
0 & 4 & 1 & 3 & 4 & 8 & 0 & 19 & 1 & 3 & 10 & 3 & 1 \\
0 & 1 & 1 & 5 & 8 & 5 & 5 & 0 & 7 & 3 & 7 & 3 & 1 \\
3 & 5 & 3 & 10 & 6 & 5 & 9 & 4 & 0 & 5 & 7 & 0 & 2 \\
0 & 8 & 0 & 0 & 7 & 5 & 7 & 6 & 6 & 0 & 3 & 0 & 0 \\
0 & 2 & 2 & 1 & 4 & 2 & 13 & 12 & 6 & 8 & 0 & 0 & 1 \\
0 & 0 & 0 & 0 & 0 & 1 & 2 & 2 & 0 & 0 & 0 & 0 & 0 \\
0 & 0 & 0 & 2 & 0 & 2 & 3 & 1 & 2 & 0 & 0 & 0 & 0
\end{array}\right) .
$$

Each element $g_{i j}$ denotes the number of passes (or attempted passes) from player $i$ to player $j$. Accordingly Messi played 19 passes to Tévez; and Rodríguez played 13 passes to Messi and 12 to Tévez, for example. It now remains to calculate the eigenvalues of G. Calculating these, we find that the largest eigenvalue is $\lambda=$ 43.1502, and the associated eigenvector is given by

$\mathbf{p}_{\lambda}=(1.517,5.074,4.113,4.222,3.202,2.127,5.09,4.216,5.483,4.242,5.197,0.481,1)$.

$\mathbf{p}_{\lambda}$ is the vector of within EVP, the individual team productivities of the players of the Argentinean players. Based on these productivities the ranking of the players is given by $(9,11,7,2,10,4,8,3,5,6,1,13,12)$. Accordingly, Mascherano (player 9) has the highest within EVP, 5.483, Rodríguez (player 11) the second highest, 5.197, and Messi (player 7) the third highest, 5.09. Note that the within EVP of Aguero (player 12) and Pastore (player 13) are quite low since they were only 15 respectively 20 minutes on the field.

In order to correct for the individual playing time, we may calculate individual team productivities on an as-if basis. To this end we extrapolate the passes of the four players who have played less than the full playing time to the total time of 90 minutes. For example, Di Maria (player 5) has played only 75 of the full 90 minutes,

\footnotetext{
${ }^{13}$ The number of passes between the Argentinean players have been provided by FAS.research, Wien, New York, www.fas.at.
} 
so we extrapolate both the passes he played and the passes he received to the total playing time by multiplying the fifth row and the fifth column of $\mathbf{G}$ by $90 / 75=1.2$; and similarly for players 10, 12 and 13. In this way, we arrive at the matrix of notional passes:

$$
\tilde{\mathbf{G}}=\left(\tilde{g}_{i j}\right)_{i, j \in T}=\left(\begin{array}{ccccccccccccc}
0 & 5 & 2 & 1 & \frac{6}{5} & 2 & 0 & 0 & 1 & \frac{18}{7} & 1 & 0 & \frac{9}{2} \\
2 & 0 & 9 & 1 & \frac{24}{5} & 5 & 6 & 1 & 9 & \frac{72}{7} & 6 & 0 & 9 \\
1 & 9 & 0 & 7 & \frac{6}{5} & 0 & 3 & 3 & 7 & \frac{9}{7} & 5 & 0 & \frac{9}{2} \\
0 & 0 & 5 & 0 & 6 & 3 & 7 & 9 & 7 & 0 & 4 & 6 & 27 \\
0 & 0 & \frac{6}{5} & \frac{12}{5} & 0 & \frac{36}{5} & \frac{66}{5} & \frac{48}{5} & 0 & \frac{162}{35} & \frac{12}{5} & 0 & 0 \\
0 & 4 & 0 & 0 & \frac{18}{5} & 0 & 5 & 1 & 2 & 0 & 4 & 6 & 0 \\
0 & 4 & 1 & 3 & \frac{24}{5} & 8 & 0 & 19 & 1 & \frac{27}{7} & 10 & 18 & \frac{9}{2} \\
0 & 1 & 1 & 5 & \frac{48}{5} & 5 & 5 & 0 & 7 & \frac{27}{7} & 7 & 18 & \frac{9}{2} \\
3 & 5 & 3 & 10 & \frac{36}{5} & 5 & 9 & 4 & 0 & \frac{45}{7} & 7 & 0 & 9 \\
0 & \frac{72}{7} & 0 & 0 & \frac{54}{5} & \frac{45}{7} & 9 & \frac{54}{7} & \frac{54}{7} & 0 & \frac{27}{7} & 0 & 0 \\
0 & 2 & 2 & 1 & \frac{24}{5} & 2 & 13 & 12 & 6 & \frac{72}{7} & 0 & 0 & \frac{9}{2} \\
0 & 0 & 0 & 0 & 0 & 6 & 12 & 12 & 0 & 0 & 0 & 0 & 0 \\
0 & 0 & 0 & 9 & 0 & 9 & \frac{27}{2} & \frac{9}{2} & 9 & 0 & 0 & 0 & 0
\end{array}\right) .
$$

The largest eigenvalue of $\tilde{\mathbf{G}}$ equals $\tilde{\lambda}=54.4199$, and the associated eigenvector is given by

$$
\mathbf{p}_{\tilde{\lambda}}=(0.401,1.255,0.968,1.431,0.86,0.514,1.396,1.216,1.4,1.16,1.261,0.633,1) .
$$

Accordingly, the ranking of the players based on the within EVP for notional passes $\mathbf{p}_{\tilde{\lambda}}$ is given by $(4,9,7,11,2,8,10,13,3,5,12,6,1)$. Thus, Heinze, Mascherano and Messi have the highest individual team productivities on an as-if basis.

\section{Conclusion}

This paper tackles a classic problem in labour economics: how to measure individual contributions to a team or a group? In the theory of teams and team production, individual contributions to the joint output of a team is referred to as individual team productivity (or co-worker productivity). Owing to the fact that a team's output is, by definition, produced jointly and frequently simultaneously, individual team productivity is both difficult to conceptualise and hard to measure. In this paper we contribute to both issues: we provide two variants of a concept of individual team productivity and show how they can be applied to given data. 
Both variants of our concept build upon eigenvector centrality, a concept well established in network analysis. Our first concept, which we call between eigenvalue productivity (between EVP), utilises the fact that different team compositions (manning schedules or occupations) and their respective success can frequently be observed. If this data is available, the relative success of any pair of team-mates can be used to measure individual team productivity of each co-worker of a given personnel or staff. Sometimes, though, there is not sufficient variation in team composition such that the concept of between EVP is not readily applicable. In order to deal with this problem, we propose a second concept to which we refer as within eigenvalue productivity (within EVP). This alternative concept rests upon bilateral co-operation between any two team-mates (or co-workers) in the course of a larger project. If temporary co-operation is observable, variations in pairwise co-operation may be used to measure individual team productivity. In both concepts, between EVP and within EVP, individual team productivities are, by construction, interdependent and must thus be calculated for all co-workers simultaneously.

Both concepts can be applied to diverse types of joint production in almost any working environment. In order to demonstrate this, we apply both concepts to team sports, and illustrate how individual productivities can be calculated from given data. If the data for the sportive success (performance) of different line-ups during a season is available, this information can be utilised to calculate each player's between EVP. If, however, a team has played only few games (for example, during a given tournament like the World Championships), there is not enough data to calculate between EVP, but within EVP might still be calculated. This can be done if, for example, data of bilateral pass statistics (directional passes played between any two players), or alternative measures of individual contributions to team success, are available. Hence, with the concepts of between and within EVP at hand, we are equipped with a method to calculate the team productivity of each player from the line-ups of the team and its performance during the season, respectively from the passes played between the players during a game.

Since either type of data is frequently available, (the two versions of) our concept can widely been used to calculate individual team productivities. For this reason, we believe that our contribution does not only represent a worthwhile piece of conceptual work, but that our concept may significantly improve empirical work in labour (and team sport) economics: As it provides a sound standing basis for the calculation of individual team productivities, the productivities calculated in 
this way may be used as dependent or explanatory variables within an econometric model. For example, the calculated individual team productivity of a player may help to explain his/her market value or salary. We are thus confident that our conceptual approach to individual team productivity will be valuable for both theoretical and empirical work in labour economics.

\section{REFERENCES}

J. Alvarez, D. Forrest, I. Sanz, and J. D. Tena. Impact of Importing Foreign Talent on Performance Levels of Local Co-workers. Labour Economics, 18(3):287-296, 2011.

Nikolaus Beck and Mark Meyer. Modeling Team Performance: Theoretical and Empirical Annotations on the Analysis of Football Data. Empirical Economics, 43:3335-356, 2012.

David J. Berri. Who Is 'Most Valuable'? Measuring the Player's Production of Wins in the National Basketball Association. Managerial and Decision Economics, 20(8):411-427, 1999.

$\mathrm{P}$ Bonacich. Factoring and Weighting Approaches to Status Scores and Clique Identification. Journal of Mathematical Sociology, 2(1):113-120, 1972.

II Depken, Craig A. Wage Disparity and Team Productivity: Evidence from Major League Baseball. Economics Letters, 67(1):87-92, 2000.

II Depken, Craig A. and Lisa E. Haglund. Peer Effects in Team Sports: Empirical Evidence from NCAA Relay Teams. Journal of Sports Economics, 12(1):3-19, 2011.

Egon Franck and Stephan Nüesch. The effect of talent disparity on team productivity in soccer. Journal of Economic Psychology, 31(2):218-229, 2010.

Felix R. Gantmacher. The Theory of Matrices, Vol. 2. AMS Chelsea Publishing, 2000.

Eric D. Gould and Eyal Winter. Interactions between Workers and the Technology of Production: Evidence from Professional Baseball. Review of Economics and Statistics, 91(1):188-200, 2009.

Roger A. Horn and Charles R. Johnson. Matrix Analysis. Cambridge University Press, 1990.

Todd Idson and Leo Kahane. Teammate Effects on Pay. Applied Economics Letters, 11(12):731-733, 2004.

Todd L. Idson and Leo H. Kahane. Team Effects on Compensation: An Application to Salary Determination in the National Hockey League. Economic Inquiry, 38 (2):345-357, 2000. 
Matthew O. Jackson. Social and Economic Networks. Princeton University Press, 2008.

Leo Katz. A New Status Index Derived from Sociometric Analysis. Psychometrika, 18(1):39-43, 1953.

Todd D. Kendall. Spillovers, Complementarities, and Sorting in Labor Markets with an Application to Professional Sports. Southern Economic Journal, 70(2): 389-402, 2003.

Alexandre Mas and Enrico Moretti. Peers at Work. American Economic Review, 99(1):112-145, 2009.

Paul Milgrom and John Roberts. The economics of modern manufacturing: Technology, strategy, and organization. American Economic Review, 80(3):511-528, 1990.

Rob Simmons and David J. Berri. Mixing the Princes and the Paupers: Pay and Performance in the National Basketball Association. Labour Economics, 18(3): 381-388, 2011.

Akira Takayama. Mathematical Economics. Cambridge University Press, 2nd edition, 1985.

Donald M. Topkis. Supermodularity and Complementarity. Princeton University Press, 1998. 